Preprint number

Date

\title{
Renormalization group study of the higher derivative conformal scalar model
}

\author{
J. A. de Barros ${ }^{a}$ 円, $\quad$ I. L. Shapiro ${ }^{a, b}$ 月 \\ a) Departamento de Fisica - ICE, Universidade Federal de Juiz de Fora, Juiz de Fora, 36036-330, \\ MG, Brazil \\ b) Tomsk State Pedagogical Institute, Tomsk, 634041, Russia
}

\begin{abstract}
The second alternative conformal limit of the recently proposed general higher derivative dilaton quantum theory in curved spacetime is explored. In this version of the theory the dilaton is transformed, along with the metric, to provide the conformal invariance of the classical action. We find the corresponding quantum theory to be renormalizable at one loop, and the renormalization constants for the dimensionless parameters are explicitly shown to be universal for an arbitrary parametrization of the quantum field. The renormalization group equations indicate an asymptotic freedom in the IR limit. In this respect the theory is similar to the well-known model based on the anomaly-induced effective action.
\end{abstract}

\section{Introduction}

The gravitational effective action, generated by the trace anomaly of the conformal invariant matter in curved spacetime, is of considerable interest due to numerous physical applications (see [1] for the review) and also because of the hope to use such an action as an insight for the theory of quantum gravity [2, 3, 4, 5, 6]. The first solution for the effective action has been obtained by Reigert [7] and by Fradkin and Tseytlin [8] (see also [9, 10, 11]). The equation solved in [7, 8] links the effective action with the trace anomaly of the Energy-Momentum tensor

$$
-\frac{2}{\sqrt{-g}} g_{\mu \nu} \frac{\delta \Gamma}{\delta g_{\mu \nu}}=T_{\mu}^{\mu}
$$

The above equation remains unaltered if one changes the solution according to $\Gamma \rightarrow \Gamma+S_{c}$, where $S_{c}\left(g_{\mu \nu}\right)$ is an arbitrary conformal invariant functional. Therefore this equation doesn't define $\Gamma\left(g_{\mu \nu}\right)$ completely, and in fact the most complicated part of the effective action remains hidden in $S_{c}\left(g_{\mu \nu}\right)$. As a result the nonconformal part of the effective action is ambiguous, and the particular version derived in [7, 8] fails to pass the test based on the calculation of the three point functions for the gravitational field [4, 12, 13. Consequently, it is important to find a solution for (1) which assumes such a test, but this problem has not been solved yet [6]. Another discrepancy arises if one compares the solution of [7, 8] with the result of direct calculations of the effective action performed in [14]. The effective action for the conformal scalar field [14] is a nonlocal functional - as well as the solution of [7, [ - but the nonlocalities are related with the Green functions of the second order conformal operator

$$
\Delta_{2}=\square-\frac{1}{6} R
$$

\footnotetext{
${ }^{1}$ Electronic address: acacio@fisica.ufjf.br

${ }^{2}$ Electronic address: shapiro@fisica.ufjf.br
} 
while in [7, 8] they are related with the Green function of the fourth order conformal operator

$$
\Delta_{4}=\square^{2}+2 R^{\mu \nu} \nabla_{\mu} \nabla_{\nu}-\frac{2}{3} R \square-\frac{1}{3} \nabla^{\mu} R \nabla_{\mu} .
$$

Indeed, any two solutions of (11) differ by the conformally invariant functional, which is supposed to be nonlocal and probably very complicated. At the same time, in many known cases the nonlocality can be removed by introducing the auxiliary scalar fields, while all the symmetries of the theory are preserved. Therefore, it is worth to study a possible forms of conformal invariant metric-dilaton models and relations between them.

In previous papers [20, 15] we have studied the renormalization and the renormalization group of the general high derivative dilaton model where we were especially interested in its conformal limit associated with the $\Delta_{4}$ operator (3). Here we will be mainly concerned and try to complete the similar program about the second (alternative) conformal invariant limit of the same general theory [15]. This alternative conformal model is based on the second order operator (2) and in this respect it is closer to the desirable form of the anomaly-induced effective action for gravity [4, 14, 6]. Our conformal theory is a direct (and quite simple) generalization of the one proposed some years ago by Antoniadis, Iliopoulos and Tomaras [16], and differs from it by the reparametrization of the scalar field [17].

The paper is organized in the following way. In the next section we write down the action of the general high derivative dilaton model and of the both of its conformal limits. We ascertain that in $D=4$ the difference between these two conformal invariant theories is the low of conformal transformation for the dilaton. Moreover, we try to generalize both theories to $n$ dimensions, $n$ different from 4 , and show that in any $n \neq 4$ the transformation low for the dilaton in two theories can be equal. Some special transformation properties of the second conformal model are also considered, and the higher derivative generalization of the conformal duality [18, 17, 19] is formulated. In section 3 the one-loop divergences for the second conformal model are derived and their parametrization dependence is studied. In the fourth section we consider the renormalization group equations for the model. The last section contains conclusion and some suggestions for the future study.

\section{General high derivative scalar model and two of its conformal limits.}

The action of the general high derivative scalar model has the form [15]

$$
\begin{aligned}
& S=\int d^{4} x \sqrt{-g}\left\{b_{1}(\square \varphi)^{2}+b_{2}(\nabla \varphi)^{2} \square \varphi+b_{3}(\nabla \varphi)^{4}+b_{4}(\nabla \varphi)^{2}+b_{5}+c_{1} R(\nabla \varphi)^{2}+\right. \\
& \left.+c_{2} R^{\mu \nu} \partial_{\mu} \varphi \partial_{\nu} \varphi+c_{3} R \square \varphi+a_{1} R_{\mu \nu \alpha \beta}^{2}+a_{2} R_{\mu \nu}^{2}+a_{3} R^{2}+a_{4} R\right\}+ \text { (surface terms). }
\end{aligned}
$$

Here the generalized couplings $a, b, c$ are some functions of the scalar field $\varphi . a, b$, and $c$ are dimensionless, except for $b_{4}, b_{5}$ and $a_{4}$, for which we have: $b_{4}(\varphi) \sim m^{2}, b_{5}(\varphi) \sim m^{4}, a_{4}(\varphi) \sim m^{2}$. All other possible terms, with an appropriate dimension which can be included into the action, differ from the ones which are already present by surface terms only [15]. We also used the notation $g^{\mu \nu} \partial_{\mu} \varphi \partial_{\nu} \varphi=(\nabla \varphi)^{2}$.

The first conformal limit of the metric-dilaton model is related with the solution of [7, 8]. Such a conformal model has been formulated in 20, 15. The action of the model is

$$
S_{c}^{(1)}=\int d^{4} x \sqrt{-g}\left\{\frac{1}{2} f(\varphi) \varphi \Delta_{4} \varphi+q(\varphi) C_{\mu \nu \alpha \beta} C^{\mu \nu \alpha \beta}+p(\varphi)(\nabla \varphi)^{4}+v E\right\},
$$

where $f, q, p$ are some differentiable functions of $\varphi, v=$ const., and $C_{\alpha \beta \mu \nu}$ is the Weyl tensor. The square of this tensor will be denoted in what follows as $C_{\mu \nu \alpha \beta} C^{\mu \nu \alpha \beta}=C^{2}$. The action includes the 
Gauss-Bonnet topological term $\int E$, where $E=R_{\alpha \beta \mu \nu}^{2}-4 R_{\alpha \beta}^{2}+R^{2}$. This term is a full derivative in four dimensions, but in $n \neq 4$ it cannot be ajusted to be conformally invariant, while $C^{2}$ can.

As was discussed in [20], the action (5) includes the term $\varphi \Delta_{4} \varphi$ which is a necessary component of the solution for the anomaly-induced effective action [7], as far as we want to write it in a local form. In 15] it was shown that, at one loop, the generic theory (5) is multiplicatively renormalizable. Moreover, the one-loop divergences has been obtained. In accordance with the general theorem [23, 24] the one-loop divergences are conformal invariant if the initial model had such invariance. In the calculations of 15] a background field method and Schwinger-DeWitt formalism in its modern form were used, and only the scalar field was considered as a quantum field, while the metric was regarded as a purely classical background. In this way, it was found that for some special choice of the conformal model, that is for some particular form of the functions $f, q, p$, the theory is one-loop finite (in the dimensional regularization) and, therefore, it is free from the conformal anomalies.

The action (5) may be written as a particular case of (4) - with special conformal constraints which have the form (below we shall frequently abandon the argument $\phi$ )

$$
a_{1}=-\frac{1}{2} a_{2}=3 a_{3}=q(\varphi), \quad b_{1}=f^{\prime}(\varphi) \varphi+f(\varphi)=\frac{3}{2} c_{1}=-\frac{1}{2} c_{2}, \quad b_{2}=b_{1}^{\prime}, \quad b_{3}=p(\varphi)
$$

while the rest of the generalized couplings $a_{4}(\varphi), b_{4}(\varphi), b_{5}(\varphi), c_{3}(\varphi)$ are equal to zero, and we use "'" to denote differentiation with respect to $\varphi$. The one-loop finite power-form solutions for $b_{1,2,3}$ found in [15] have the form (with accuracy to an overall constant):

$$
\begin{gathered}
b_{1}=1, \quad b_{2}=0, \quad b_{3}=0, \\
b_{1}=1, \quad b_{2}=b_{1}{ }^{\prime}=0, \quad b_{3}=3 / 5\left(\varphi-\varphi_{0}\right)^{-2}, \quad \varphi_{0}=\text { const. }, \\
b_{1}=\left(\varphi-\varphi_{0}\right)^{-2}, \quad b_{2}=b_{1}{ }^{\prime}=-2\left(\varphi-\varphi_{0}\right)^{-3}, \quad b_{3}=\left(\varphi-\varphi_{0}\right)^{-4} .
\end{gathered}
$$

In the second solution (8) $b_{3}$ can be generalized to $b_{3}=F^{-1}\left(\varphi-\varphi_{0}\right)$ where the function $F(p)$ is the solution of the differential equation $p^{\prime \prime}-10 p^{2}=0$. This solution is independent on the first one (7), but one can easily see that the third one (9) coincides with (7) after a nonpolinomial reparametrization $\varphi \rightarrow \ln \varphi$ in the former. Thus, we have only two independent solutions. This example shows the importance of tracing an arbitrary parametrization of the background field in the sigma-model type theories in $n=4$. The parametrization of the quantum field is also important, since it can modify the renormalization constants and therefore the beta-functions and scaling behaviour. A comprehensive formal study of the parametrization dependence in quantum field theory can be found in [21]. However, explicit loop calculations in an arbitrary parametrization of the quantum field should also be interesting. In the next section we investigate in details the parametrization dependence for the one-loop divergences of the second conformal high derivative scalar model (10).

The model (5) is invariant under a local conformal transformation of the metric only $\bar{g}_{\mu \nu}=$ $g_{\mu \nu} e^{2 \sigma(x)}$, while the dilaton is not transformed. However, in (画 the transformation rule for the dilaton is not fixed, and that is why there exists the second conformal limit of (4) with the nontrivial transformation rule for the dilaton [17].

$$
S_{c}^{(2)}=\int d^{4} x \sqrt{-g}\left\{v E+x C^{2}+y\left[\frac{R}{3}-\frac{\square \zeta}{\zeta}+\frac{(\nabla \zeta)^{2}}{2 \zeta^{2}}\right]^{2}+\frac{3 z \zeta}{\gamma}\left[\frac{R}{3}-\frac{\square \zeta}{\zeta}+\frac{(\nabla \zeta)^{2}}{2 \zeta^{2}}\right]-\frac{u \zeta^{2}}{\gamma^{2}}\right\},
$$

where $\zeta=\zeta(\phi)$ is the function of the scalar field $\phi, \gamma$ is (dimensional) Newton's constant, and $x, y, z, u$ are some dimensionless constants. The last action is invariant under the transformation 
which includes an arbitrary reparametrization of the scalar field and the special conformal transformation of the metric

$$
\bar{\phi}=\bar{\phi}(\phi), \quad \bar{g}_{\mu \nu}=g_{\mu \nu}\left[\frac{\zeta(\phi)}{\zeta(\bar{\phi}(\phi))}\right] .
$$

One has to notice that the reparametrization of the scalar field alone, without the transformation of the metric, doesn't change neither the form of the action (10) nor the values of the constants $\gamma, v, x, y, z, u$. It only changes the form of the function $\zeta(\phi)$. In this way one can cast the action (10) into the form originally proposed by Antoniadis, Iliopoulos and Tomaras [16]

$$
S_{c}=\int d^{4} x \sqrt{-g}\left\{v E+x C^{2}+y\left(\phi^{-1} \Delta_{2} \phi\right)^{2}-\frac{6 z}{\gamma} \phi \Delta_{2} \phi-u \phi^{4}\right\} .
$$

In general, the new action - with another parametrization of the scalar field - will possess the same symmetry (11) as the original one. Therefore the reparametrization of the dilaton in (10) transfers one such theory into another, with different form of $\zeta(\phi)$. The same result can be achieved by doing the conformal transform of the metric in (11) without affecting the dilaton. Moreover, the conformal transformation of the metric can do what reparametrization of the scalar cannot - it can make the new $\zeta$ constant. As a result one arrives at the familiar action for the higher derivative gravity (see (22, 24] and references therein)

$$
S_{H D}=\int d^{4} x \sqrt{-\bar{g}}\left\{v E+x C^{2}+y \bar{R}^{2}+\frac{z}{3 \gamma} \bar{R}-\frac{u}{\gamma^{4}}\right\}
$$

with all $v, x, y, z, u, \gamma$ constants. Thus, the transformation properties of (10) are quite similar to the ones of the second derivative conformal metric-dilaton theory considered in [17]. The difference is that the second derivative model is conformally equivalent to the Einstein gravity, whereas the fourth derivative model (10) to the higher derivative gravity (13). In both cases an extra conformal symmetry "eats" the extra scalar field. Of course the second derivative sector of (10) reproduces the model of [17] literally.

Let us mention that one can easily formulate the conformal duality, which takes place in the second derivative metric-dilaton models [18, 17, 19], for its fourth derivative cousin?]. To see this we denote the action (10) as $S_{\zeta(\phi) ; v, x, y, z, u}$ and, following the method of [17], construct a sum

$$
S_{\zeta(\phi) ; v, x, y, z, u}\left(\phi, g_{\mu \nu}\right)+S_{\xi(\phi) ; v^{\prime}, x^{\prime}, y^{\prime}, z^{\prime}, u^{\prime}}\left(\phi, g_{\mu \nu}\right)
$$

where $v^{\prime}, x^{\prime}, y^{\prime}, z^{\prime}, u^{\prime}$ are some other constants and $\xi(\phi)$ - some other function. If we perform the transformation of the metric $g_{\mu \nu}=\bar{g}_{\mu \nu} \Omega(\phi)$ and take $\Omega=N(\phi) / \zeta(\phi)$, with a differentiable function $N(\phi)$, then (14) becomes

$$
S_{N(\phi) ; x, y, z, u}\left(\phi, \bar{g}_{\mu \nu}\right)+S_{\frac{N(\phi) \xi(\phi)}{\zeta(\phi)} ; x^{\prime}, y^{\prime}, z^{\prime}, u^{\prime}}\left(\phi, \bar{g}_{\mu \nu}\right) .
$$

In particular, for $\zeta=\phi^{2}$ and $\xi=\kappa^{2}=$ const we meet the symmetry which is a direct generalization of the conformal duality of [18, 17, 19]:

$$
\phi \longleftrightarrow \frac{1}{\phi}, \quad \kappa \longleftrightarrow \frac{1}{\kappa}, \quad(x, y, z, u) \longleftrightarrow\left(x^{\prime}, y^{\prime}, z^{\prime}, u^{\prime}\right), \quad g_{\mu \nu} \longleftrightarrow \bar{g}_{\mu \nu}=\frac{\zeta}{N} g_{\mu \nu}
$$

One can formulate this dual symmetry in any spacetime dimension different than 2 , as it was done in 19] with the second derivative metric-dilaton model.

\footnotetext{
${ }^{3}$ Another form of conformal duality takes place in some purely metric theories with action $\int d^{4} x \sqrt{-g} L(R)$ 25. Such models can be reduced to the second derivative metric-dilaton theory 26]. The underlying metric-dilaton model is associated with the operator (2) and in this sense can be close to the one considered in [18, 17].
} 
As we have seen, there are two conformal limits in the general model (雨), the only difference between them is a different rule of transformation for the dilaton. As far as in quantum domain we suppose to use the dimensional regularization, it is significant that one can, in some extent, formulate the conformal invariant models of both types in a spacetime dimension different than 4 . To construct a version of (间) in arbitrary $n$ we have to use the $n$-dimensional generalization of the fourth-order conformally-covariant self-adjoint operator $\Delta_{4}$ (3). The $\Delta_{4}$ operator was originally invented in an arbitrary dimension [27] (see also [28, 30] for the wider classes of conformal operators and 29] for the recent investigation of $\Delta_{4}$ ), where it has the form

$$
\begin{aligned}
\Delta_{4}^{(n)}= & \square^{2}+\frac{4}{n-2}\left[R^{\mu \nu} \nabla_{\mu} \nabla_{\nu}+\frac{1}{2}\left(\nabla^{\mu} R\right) \nabla_{\mu}\right]+\frac{4 n-n^{2}-8}{2(n-1)(n-2)}\left[R \square+\left(\nabla^{\mu} R\right) \nabla_{\mu}\right]+ \\
& +(4-n)\left[\frac{1}{4(n-1)}(\square R)+\frac{8-5 n}{16(n-1)^{2}(n-2)} R^{2}+\frac{1}{(n-2)^{2}} R_{\mu \nu} R^{\mu \nu}\right] .
\end{aligned}
$$

The last term in (5) cannot be conformally continued to $n \neq 4$ and hence the corresponding action has the form

$$
S_{c}^{(1, n)}=\int d^{n} x \sqrt{-g}\left\{\frac{1}{2} f \varphi \Delta_{4}^{(n)} \varphi+q \varphi^{2} C^{2}\right\}
$$

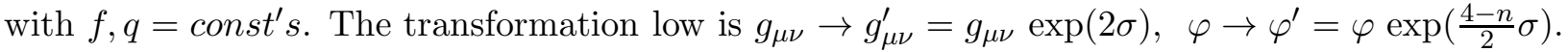
For $n=4$ the above action is reduced to a particular case of (5). Indeed there is the possibility of an arbitrary reparametrization of the scalar (just as in (5), if we do so, then the form of the conformal transformation should be modified in the spirit of (11)). We remark, that the pure Weyl gravity is nonconformal in $n \neq 4$, and the scalar field $\varphi$ (with a nontrivial transformation low) serves as a compensator which provides the invariance of the $C^{2}$-term.

The $n$-dimensional form of (10) can be easily written for the Antoniadis, Iliopoulos and Tomaras theory (12). As we already mentioned above, this version differs from the general one (10) by the reparametrization of the scalar, so one can easily rewrite the result for the case of (10). In the $n$-dimensional case the conformal invariant action is

$$
S_{c}=\int d^{n} x \sqrt{-g}\left\{x \phi^{\frac{2(n-4)}{n-2}} C^{2}+y \phi^{\frac{2(n-4)}{n-2}}\left(\phi^{-1} \Delta_{2}^{(n)} \phi\right)^{2}-\frac{6 z}{\gamma} \phi \Delta_{2}^{(n)} \phi-u \phi^{\frac{2 n}{n-2}}\right\},
$$

where the operator $\Delta_{2}^{(n)}$ and the transformation rules have usual form (see, for example, [24])

$$
\Delta_{2}^{(n)}=\square+\frac{n-2}{4(n-1)} R, \quad g_{\mu \nu} \rightarrow g_{\mu \nu}^{\prime}=g_{\mu \nu} e^{2 \sigma}, \quad \phi \rightarrow \phi^{\prime}=\phi e^{\frac{n-2}{2} \sigma} .
$$

The relevant point is that after the reparametrization of the scalar $\phi=\chi^{\frac{4-n}{2-n}}$ the form of the action (19) changes, and the transformation rule for the new field $\chi$ is exactly the same as for the scalar field $\varphi$ of the theory (5). Indeed, the above reparametrization becomes degenerate at $n=4$. Thus in any dimension $n \neq 4$ there are two different conformal models (19) and (18) which include the second $\Delta_{2}^{(n)}$ and the fourth $\Delta_{4}^{(n)}$ order operators in the dilaton sector. For any $n \neq 4$, with accuracy to the parametrization of the scalar field, the field content and symmetries of the theories are exactly the same. However we fail to see the transformation which links two conformal models. On the other hand, another kind of relation between them arises if we consider both models as a one-loop renormalizable versions of the general high derivative theory (4). We shall discuss quantum aspects of the theory in the next section.

\section{Renormalization of second conformal model in arbitrary parametrization.}

The purpose of this section is to investigate the one-loop renormalization of the theory (10). As we already know, for the classical theory the form of the function $\zeta(\phi)$ in the action can be 
changed by reparametrizing the scalar field. If we intend to study the one-loop effective action in the framework of the background field method, then the reparametrization of the background field doesn't create anything new with respect to the classical theory. On the other hand, the reparametrization of the quantum field can essentially affect the one-loop divergencies, so that only the on-shell quantities remain invariant In our case, due to its simplicity, the theory (10) gives an opportunity to trace the off-shell dependence on the parametrization of the quantum field explicitly in a general form (we remind that only the scalar is a quantum field). To do this one has to write the action (10) as the particular form of (4) and then use the result for the one-loop divergences of the last theory derived in 15]. The problem is to find an explicit form of the renormalization for the function $\zeta(\phi)$ and for the parameters $v, x, y, z, u$, which removes the counterterms in an arbitrary parametrization.

The conformal invariant action (10) corresponds to the following constraints for the functions $a_{i}(\phi), b_{j}(\phi), c_{k}(\phi)$ of the model (雨), where we put $\gamma=1$ for simplicity.

$$
\begin{gathered}
b_{1}=\frac{y\left(\zeta^{\prime}\right)^{2}}{\zeta^{2}}, \quad b_{2}=\frac{y \zeta^{\prime}\left(2 \zeta \zeta^{\prime \prime}-\left(\zeta^{\prime}\right)^{2}\right)}{\zeta^{3}}, \quad b_{3}=y \frac{4 \zeta^{2}\left(\zeta^{\prime \prime}\right)^{2}+\left(\zeta^{\prime}\right)^{4}-4 \zeta \zeta^{\prime \prime}\left(\zeta^{\prime}\right)^{2}}{4 \zeta^{4}} \\
b_{4}=\frac{3}{2} \frac{z\left(\zeta^{\prime}\right)^{2}}{\zeta}, \quad b_{5}=t \zeta^{2}, \quad c_{1}=y \frac{\left(\zeta^{\prime}\right)^{2}-2 \zeta \zeta^{\prime \prime}}{3 \zeta^{2}}, \quad c_{2}=0, \quad c_{3}=-\frac{2}{3} \frac{y \zeta^{\prime}}{\zeta} \\
a_{1}=x+v, \quad a_{2}=2 x-4 v, \quad a_{3}=-\frac{1}{3} x+v+\frac{y}{9}, \quad a_{4}=z \zeta .
\end{gathered}
$$

The one-loop counterterms for the general model (4) have the form:

$$
\begin{gathered}
\Delta S=\frac{\mu}{\varepsilon}^{n-4} \int d^{n} x \sqrt{-g}\left\{A_{1} R_{\alpha \beta \mu \nu}^{2}+A_{2} R_{\alpha \beta}^{2}+A_{3} R^{2}+A_{4} R+C_{1} R(\nabla \varphi)^{2}+C_{2} R^{\alpha \beta} \partial_{\alpha} \varphi \partial_{\beta} \varphi+\right. \\
\left.+C_{3} R(\square \varphi)+B_{1}(\square \varphi)^{2}+B_{2}(\nabla \varphi)^{2}(\square \varphi)+B_{3}(\nabla \varphi)^{4}+B_{4}(\nabla \varphi)^{2}+B_{5}\right\},
\end{gathered}
$$

where $\varepsilon=(4 \pi)^{2}(n-4)$ and cumbersome explicit expressions for the coefficients $A_{i}, B_{j}, C_{k}$ can be found in 15]. After substituting the constraints (21) into (22) we obtain the following expressions for the counterterms.

$$
\begin{gathered}
B_{1}=\frac{2\left(\zeta^{\prime \prime}\right)^{2}}{\left(\zeta^{\prime}\right)^{2}}-\frac{\zeta^{\prime \prime}}{\left(\zeta^{\prime}\right)^{2} \zeta}-\frac{\zeta^{\prime \prime \prime}}{\zeta^{\prime}}+\frac{5}{4} \frac{\left(\zeta^{\prime}\right)^{2}}{\zeta^{2}} \\
B_{2}=\frac{5 \zeta^{\prime \prime} \zeta^{\prime \prime \prime}}{\left(\zeta^{\prime}\right)^{2}}-\frac{5 \zeta^{\prime \prime \prime}}{2 \zeta}+\frac{3\left(\zeta^{\prime \prime}\right)^{2}}{\zeta^{\prime} \zeta}+\frac{2 \zeta^{\prime}}{\zeta^{2}}-\frac{\zeta^{\prime \prime \prime \prime}}{\zeta^{\prime}}-\frac{5\left(\zeta^{\prime}\right)^{3}}{4 \zeta^{3}}-\frac{4\left(\zeta^{\prime \prime}\right)^{3}}{\left(\zeta^{\prime}\right)^{3}} \\
B_{3}=\frac{1}{16}\left\{\frac{-96 \zeta^{\prime \prime \prime} \zeta^{\prime \prime}}{\zeta^{\prime} \zeta}-\frac{16 \zeta^{\prime \prime} \zeta^{\prime \prime \prime \prime}}{\left(\zeta^{\prime}\right)^{2}}+\frac{24 \zeta^{\prime} \zeta^{\prime \prime \prime}}{\zeta^{2}}+\frac{8 \zeta^{\prime \prime \prime \prime}}{\zeta}-\frac{36\left(\zeta^{\prime \prime}\right)^{2}}{\zeta^{2}}\right. \\
\left.+\frac{5\left(\zeta^{\prime}\right)^{4}}{\zeta^{4}}-\frac{12 \zeta^{\prime \prime}\left(\zeta^{\prime}\right)^{2}}{\zeta^{3}}+\frac{128\left(\zeta^{\prime \prime}\right)^{3}}{\left(\zeta^{\prime}\right)^{2} \zeta}+\frac{96 \zeta^{\prime \prime \prime}\left(\zeta^{\prime \prime}\right)^{2}}{\left(\zeta^{\prime}\right)^{3}}-\frac{96\left(\zeta^{\prime \prime}\right)^{4}}{\left(\zeta^{\prime}\right)^{4}}\right\} \\
B_{4}=\frac{3}{8} z\left(\frac{8 \zeta^{2}\left(\zeta^{\prime \prime}\right)^{2}-6 \zeta \zeta^{\prime \prime}\left(\zeta^{\prime}\right)^{2}-4 \zeta^{\prime} \zeta^{\prime \prime \prime} \zeta^{2}-3\left(\zeta^{\prime}\right)^{4}}{y\left(\zeta^{\prime}\right)^{2} \zeta}\right)
\end{gathered}
$$

\footnotetext{
${ }^{4}$ The higher loop contributions can depend on the parametrization in a more complicated way, because in this case one has to take into account the quantum corrections to the equations of motion [21, 24.

${ }^{5}$ The expression for $B_{4}$ in [15] contains a misprint. The correct form is

$$
B_{4}=-\frac{b_{4}^{\prime \prime}}{2 b_{1}}+\frac{1}{2 b_{1}^{2}}\left(4 b_{1}^{\prime \prime} b_{4}-4 b_{2}^{\prime} b_{4}+6 b_{3} b_{4}+5 b_{1}^{\prime} b_{4}^{\prime}-3 b_{4}^{\prime} b_{2}\right)+\frac{1}{b_{1}^{3}}\left(3 b_{1}^{\prime} b_{2} b_{4}-4\left(b_{1}^{\prime}\right)^{2} b_{4}\right)
$$
}




$$
\begin{gathered}
B_{5}=\frac{9}{8} \zeta^{2}\left(\frac{z^{2}}{y^{2}}-\frac{8 t\left(\zeta^{\prime}\right)^{2}+8 t \zeta \zeta^{\prime \prime}}{y\left(\zeta^{\prime}\right)^{2}}\right), \\
C_{1}=\frac{5\left(\zeta^{\prime}\right)^{2}}{12 \zeta^{2}}-\frac{5\left(\zeta^{\prime \prime}\right)^{2}}{3 \zeta^{2}\left(\zeta^{\prime}\right)^{2}}-\frac{\zeta^{\prime \prime}}{2 \zeta}+\frac{\zeta^{\prime \prime \prime}}{\zeta^{2} \zeta^{\prime}}+\frac{\zeta \zeta^{\prime \prime \prime \prime}}{3\left(\zeta^{\prime}\right)^{2}}-\frac{2 \zeta \zeta^{\prime \prime} \zeta^{\prime \prime \prime}}{\left(\zeta^{\prime}\right)^{3}}+\frac{2 \zeta\left(\zeta^{\prime \prime}\right)^{3}}{\left(\zeta^{\prime}\right)^{4}} \\
C_{2}=0, \quad C_{3}=\frac{1}{6}\left\{\frac{2 \zeta^{\prime \prime}}{\zeta^{\prime}}-\frac{5 \zeta^{\prime}}{\zeta}+\frac{2 \zeta^{\prime \prime \prime} \zeta}{\left(\zeta^{\prime}\right)^{2}}-\frac{4 \zeta\left(\zeta^{\prime \prime}\right)^{2}}{\left(\zeta^{\prime}\right)^{3}}\right\}, \\
A_{1}=\frac{1}{90}, \quad A_{2}=-\frac{1}{90}, \quad A_{3}=\frac{5}{36}, \quad A_{4}=-\frac{z \zeta\left(2 \zeta \zeta^{\prime \prime}+3\left(\zeta^{\prime}\right)^{2}\right)}{4 y\left(\zeta^{\prime}\right)^{2}} .
\end{gathered}
$$

According to general theorems [21] (see also [24]), the above formulas have to meet two requirements:

i) The coefficients $A_{i}, B_{j}, C_{k}$ satisfy the conformal constraints (21).

ii) The divergences of the renormalized action $S+\Delta S$ can be removed by the renormalization of the function $\zeta$ and of the constant parameters $x, y, z, u$.

Indeed, the transformation of the function $\zeta_{0}=\zeta+(1 / \varepsilon) f(\zeta, \phi)$ can be always solved with respect to $\phi$ with accuracy to $\mathcal{O}(1 / \varepsilon)$, and therefore the renormalization of $\zeta$ is nothing but another form of the renormalization of the field $\phi_{0}=\phi+\frac{1}{\varepsilon} f(\zeta) / \zeta^{\text {prime }}$. Of course, for general $\zeta$ such a renormalization can be nonlinear and quite complicated. The same transformation can be viewed from a different point of view. As we already know from the previous section, any change of parametrization for the scalar field doesn't violate the conformal invariance. It only modifies the form of the conformal transformation (11). Therefore, i) follows from ii), and we have to check only the former.

The search of the renormalization transformation ii) is not easy because of the complicated form of the expressions (23). That is why we shall construct such a transformation in two steps. First we take a simple particular form of $\zeta=\phi^{\alpha}$, and find the form of the renormalization constants for the parameters $v, x, y, z, u$. After that we find an explicit form of the renormalization for the function $\zeta(\phi)$.

For the special case $\zeta=\phi^{\alpha}$ the expressions (23) have the following form. First,

$$
\left(B_{1}, B_{2}, B_{3}, C_{1}, C_{2}, C_{3}\right)=\frac{5}{4 y}\left(b_{1}, b_{2}, b_{3}, c_{1}, c_{2}, c_{3}\right),
$$

while $b_{1}, b_{2}, b_{3}, c_{1}, c_{2}, c_{3}$ can be all restored from the constraints (21). Say, $b_{1}=y \alpha^{2} \phi^{-2}$ etc. This result indicates that for this special parametrization the conformal invariance in the high derivative sector of the model is preserved, that the renormalization of the function $\zeta(\phi)$ is not necessary, and that in the high derivative sector the renormalization constant for $y$ is parametrization independent. The last property have to hold for the general parametrization too. Since the relation between the above parameters is fixed, it is sufficient to consider the renormalization of one parameter, for example $b_{1}$. All others will renomalize identically. For the second- and zero-derivative sectors of the model we find

$$
\begin{gathered}
a_{4}=z \phi^{\alpha}, \quad b_{4}=\frac{3}{2} \alpha^{2} \phi^{-2} a_{4}, \quad b_{5}=u \phi^{2 \alpha}, \\
A_{4}=-\frac{1}{4} \frac{z}{y}\left(5-\frac{2}{\alpha}\right) \phi^{\alpha}, \quad B_{4}=\frac{3}{2} \alpha^{2} \phi^{-2} A_{4}, \quad B_{5}=\left(\frac{1}{\alpha} \frac{u}{y}-2 \frac{u}{y}+\frac{9}{8} \frac{z^{2}}{y^{2}}\right) \phi^{2 \alpha} .
\end{gathered}
$$

The last formula indicates that the lower order counterterms are conformal invariant, and the relation between $A_{4}$ and $B_{4}$ is fixed. All the counterterms can be removed by the renormalization of the parameters $p=(v, x, y, z, u)$

$$
p_{0}=\mu^{n-4}\left(p+\frac{F_{p}}{\varepsilon}\right)
$$


One can perform an additional renormalization of the scalar $\phi_{0}=\phi-\frac{1}{\varepsilon} \frac{\beta}{y}$ with an arbitrary $\beta$, and similar (global) renormalization of the metric. As we know from the previous section, the effect of such renormalization is the same as the one for the scalar field, but we include this transformation $g_{\mu \nu}^{0}=g_{\mu \nu}\left(1-\frac{1}{\varepsilon} \frac{\gamma}{y}\right)$, with an arbitrary parameter $\gamma$, for the sake of completeness.

Taking all this into account, the renormalization parameters become

$$
\begin{aligned}
& F_{v}=-\frac{1}{180},, \quad F_{x}=\frac{1}{60}, \quad F_{y}=-\frac{5}{4}, \\
& F_{z}=\left(\frac{1}{2 \alpha}-\frac{5}{4}+\alpha \beta+\gamma\right) \frac{z}{y}, \quad F_{u}=\left(\frac{1}{\alpha}-2+2 \alpha \beta+2 \gamma\right) \frac{u}{y}+\frac{9}{8} \frac{z^{2}}{y^{2}} .
\end{aligned}
$$

One can see that the renormalization constants for $v, x, y$ don't, but the renormalization constants for $z$ and $u$ do depend on the function $\zeta$, that is on the parametrization of the quantum scalar field. The two quantities $F_{z}, F_{u}$ depend also on an arbitrary parameters $\beta, \gamma$. One can, indeed, choose $\beta$ and $\gamma$ in such a way that both $F_{z}, F_{u}$ become independent on $\alpha$. For example, this can be achieved

for $\beta=-\frac{1}{2 \alpha^{2}}, \gamma=0$. However this doesn't mean that we are able to provide the unambiguous renormalization of the individual parameters $z, u$, because such a choice of $\beta, \gamma$ is not better than any other one. The crucial observation is that the combination $2 u F_{z}-z F_{u}$ is parametrization independent. This combination is nothing but the renormalization parameter for $\frac{z^{2}}{t}$ which is an essential coupling of the theory.

Now we are in the position to study the one-loop renormalization of the theory (10) in an arbitrary parametrization. Taking into account the renormalization of $y$ in (26), (27) we were able to establish the transformation for $\zeta(\phi)$ which, together with the "high-derivative" part of (26) and (27), provides the finiteness in the corresponding sector of the theory.

$$
\zeta^{(0)}=\mu^{n-4}\left\{\zeta-\frac{1}{2 \varepsilon} \zeta^{\prime \prime}\left(\frac{\zeta^{\prime}}{\zeta}\right)^{2}\right\} .
$$

Direct substitution shows that the above transformation, together with the appropriate renormalization of $v, x, y, z, u$ removes all the counterterms. The renormalization of the parameters $v, x, y, z, u$ has the form (26), (27),(28) with any particular values which satisfy $\gamma+\alpha \beta=\frac{\alpha-1}{2 \alpha}$, . Indeed, this doesn't mean that the renormalization of the parameters $z, u$ is independent on the parametrization of the quantum field. To ensure in the opposite it is enough to remind the results for the particular parametrization $\zeta=\phi^{\alpha}$, which we have discussed above. Only the renormalization of the dimensionless ratio $u / z^{2}$ is invariant and unique.

Thus, the theory under consideration is one-loop renormalizable. Contrary to the first conformal model (5) explored in [15], our model (10) doesn't allow finite solutions. This follows directly from the existence of the parameters $y$ and $u / z^{2}$, which have universal and non-zero renormalization. Therefore, in this model one always meets the conformal anomaly, which modifies the finite part of the effective action, and breaks the conformal symmetry.

\section{Renormalization group equations}

In the previous section we have seen that the theory (10) is one-loop renormalizable, so we can use the renormalization group method to investigate its scaling behaviour. Here we regard (10) as a theory of quantum scalar field in curved spacetime, and therefore the renormalization group should also be formulated in curved spacetime (one can look at 24] for the introduction and references on the renormalization group in an external gravitational field). The renormalization group equation for the effective action has the following solution,

$$
\Gamma\left[e^{-2 t} g_{\mu \nu}, \phi, p, \mu\right]=\Gamma\left[g_{\mu \nu}, \phi(t), p(t), \mu\right],
$$


which enables one to explore the short-distance (and therefore high-energy) limit, which we shall call UV. In the massless conformal theories the same equation can serve for the investigation of the longdistance (IR) limit. The scaling behaviour of the effective charges $p(t)=(x(t), y(t), z(t), u(t), v(t))$ is related to the conventional $\beta$-functions.

$$
\beta_{p}(n)=\mu \frac{d p}{d \mu},
$$

where the limit $\beta_{p}=\lim _{n \rightarrow 4} \beta_{p}(n)$ should be taken after the operation (31) over the renormalized parameter $p$. One can construct also the renormalization group equations for $\phi(t)$ and $g_{\mu \nu}(t)$ but they will be ambiguous, and we do not write them here. For the sake of simplicity we present the expressions for the $\beta$-functions for the spacial parametrization $\zeta=\phi^{\alpha}$. As it was demonstrated in the previous section, the renormalization of the scalar field and metric depend on the arbitrary parameters $\beta$ and $\gamma$ (28). Therefore the corresponding renormalization group equations do not have independent physical sense and we write them only in order to illustrate the parametrization dependence ?. The renormalization group equations for $x(t), y(t), z(t), u(t), v(t)$ have the form

$$
\begin{gathered}
(4 \pi)^{2} \frac{d v}{d t}=-\varepsilon v+\frac{1}{180}, \quad v(0)=v_{0}, \\
(4 \pi)^{2} \frac{d x}{d t}=-\varepsilon x-\frac{1}{60}, \quad x(0)=x_{0}, \\
(4 \pi)^{2} \frac{d y-1}{d t}=\varepsilon y^{-1}+\frac{5}{4} y^{-2}, \quad y(0)=y_{0}, \\
(4 \pi)^{2} \frac{d z}{d t}=-\varepsilon z+\left(\frac{5}{4}-\frac{1}{2 \alpha}-\alpha \beta-\gamma\right) \frac{z}{y}, \quad z(0)=z_{0}, \\
(4 \pi)^{2} \frac{d u}{d t}=-\varepsilon u+\left(2-\frac{1}{\alpha}-2 \alpha \beta-2 \gamma\right) \frac{u}{y}-\frac{9}{8} \frac{z^{2}}{y^{2}}, \quad u(0)=u_{0},
\end{gathered}
$$

where $\varepsilon=(4 \pi)^{2}(n-4)$. We have written the renormalization group equation for $y^{-1}$ because just this quantity is the parameter of the loop expansion in the path integral, so the behaviour of $y^{-1}$ in UV and IR limits defines the asymptotical properties of the theory. The equations for the individual $z(t), u(t)$ are ambiguous, so that one can define uniquely only the behaviour of the essential coupling constant $\lambda=\frac{u}{z^{2}}$, for which we have

$$
(4 \pi)^{2} \frac{d \lambda}{d t}=\varepsilon \lambda-\frac{1}{2 y} \lambda-\frac{9}{8 y^{2}}, \quad \lambda(0)=\frac{u_{0}}{z_{0}^{2}}
$$

The solution of the above equations is straightforward. We present the solutions for $n=4$.

$$
\begin{gathered}
x(t)=x_{0}-\frac{1}{60(4 \pi)^{2}} t, \quad v(t)=v_{0}+\frac{1}{180(4 \pi)^{2}} t, \quad y^{-1}(t)=y_{0}^{-1}\left(1-\frac{5}{4(4 \pi)^{2}} y_{0}^{-1} t\right)^{-1}, \\
\lambda(t)=\left(\lambda_{0}+\frac{9}{14 y_{0}}\right)\left(\frac{y(t)}{y_{0}}\right)^{\frac{2}{5}}-\frac{9}{14 y(t)} .
\end{gathered}
$$

The solution for $y(t)$ indicates that the theory (10) is asymptotically free in IR. The formulas for $x(t), v(t)$ describe linear variations of these effective charges with scale. The last solution gives the evolution of the cosmological constant measured in units of the Newtonian constant (or v.v.). For the spacial initial conditions of the renormalization group flow $\lambda_{0}=-\frac{9}{14 y_{0}}$ we meet the decreasing

\footnotetext{
${ }^{6}$ This high degree (two-parametric) of ambiguity is in fact caused by the conformal (global) invariance of the theory, it can be weakened if one introduces the mass of the scalar field or Einstein term.
} 
cosmological constant at low energies. We remark that the behaviour of the cosmological $u$ and gravitational $z$ constants is ambiguous, as it always happens. In our case this ambiguity is caused by the freedom to change quantum variables - reparametrizations in the scalar sector - or by the freedom to reparametrize the conformal factor of the metric.

\section{Conclusions}

We have investigated some classical and quantum aspects of the second (alternative) conformal limit of the recently proposed general higher derivative dilaton quantum theory in curved spacetime. In particular, the form of conformal transformation for two conformal metric-dilaton models with

fourth derivatives has been compared, and it was shown that in the spacetime dimension $n \neq 4$ the transformations lows are identical with accuracy to the reparametrization in the scalar sector. We also generalize the conformal duality of [18, 17, 19] for the high derivative metric-dilaton theories.

The quantum theory of dilaton on an arbitrary metric background was investigated. Using the result of our earlier calculations [15] it was demonstrated that the second conformal invariant model is multiplicatively renormalizable in one loop, while for the first conformal limit the same is known from 15]. The renormalization of the dimensionless parameters was shown to be universal for an arbitrary parametrization of the quantum field. The simplicity of the models enables one to trace such a parametrization dependence explicitly, thus we find a compensating transformation for the background field. The renormalization group equations for the essential coupling constants indicate an asymptotic freedom in the IR limit. In this respect, the theory is similar to the well-known model of Antoniadis and Mottola [3], based on the anomaly-induced effective action.

In order to outline the possible directions for future studies, we can mention the possibility to investigate the renormalization, renormalization group, and specially the renormalization group improved effective potential for the theory with conformal duality (15). This is a potentially important problem, because the conformal duality can link the regimes of strong and weak gravitational field, and, therefore, we may hope to get an information about the effective potential in the vicinity of the singularities of a black hole and Big-Bang solutions. Indeed the relevance of such investigation showld be considerably higher if we would be able to make the calculations in the full theory with a quantum metric and scalar. The method of calculation in such a theory is known [20], but the actual computations seems, at the moment, unfeasable to us, due to its algebraic complexity. Alternatively, we can use some approximate methods like the $1 / N$ expansion, adding to the original theory a new set of $N$ conformal matter fields. Another interesting possibility is to solve the equation (11) with the anomalous trace of the energy-momentum tensor which corresponds to the higher derivative conformal scalar models (5) and (10). The r.h.s. of (1) is proportional to the one-loop divergences for this theories [31], which are all known from [15] and the present paper. The corresponding solution of (1) gives the finite quantum correction to the action of the theory. Therefore, it can be very interesting, in view of possible cosmological applications, because for conformally flat cosmological metrics the integration constant for (11) is irrelevant. One can mention, for instance, that a similar solution with torsion leads to inflationary model [9]. We hope to investigate the above problems in the close future.

\section{Acknowledgements}

One of the authors (I.Sh.) is grateful to the Departamento de Fisica, UFJF for warm hospitality. The work of I.Sh. was supported in part by Russian Foundation for Basic Research under the project No.96-02-16017. J.A.B. thanks FAPEMIG (Minas Gerais State Sponsoring Agency) for finantial support. 


\section{References}

[1] M.J. Duff, Class.Quant.Grav. 11, 1387 (1994).

[2] S.D. Odintsov and I.L. Shapiro, Class. Quant. Grav. 8 L57 (1991).

[3] I. Antoniadis and E. Mottola, Phys. Rev. 45D, 2013 (1992).

[4] S. Deser and A. Schwimmer, Phys.Lett. 309B 279 (1993).

[5] I.L. Shapiro and G. Cognola, Phys.Rev. 51D 2775 (1995).

[6] S. Deser, Helv.Phys.Acta 69 (1996) 570, hep-th/9609138.

[7] R.Y. Reigert, Phys.Lett. 134B, 56 (1984).

[8] E.S. Fradkin and A.A. Tseytlin, Phys.Lett. 134B, 187 (1984).

[9] I.L. Buchbinder, S.D. Odintsov and I.L. Shapiro, Phys.Lett. 162B, 92 (1985).

[10] I.L. Buchbinder, V.P. Gusynin and P.I. Fomin, Yad. Fiz. (Sov. J. Nucl. Phys.) 44, 828 (1986).

[11] J.S. Dowker, Phys.Rev. D33, 3150 (1986).

[12] H. Osborn and A. Petkou, Ann.Phys. 231 (1994) 311.

[13] J. Erdmenger and H. Osborn, Nucl.Phys. 483 (1996) 431.

[14] G.A. Vilkovisky, Class.Quant.Grav. 9895 (1992); A.O. Barvinsky, Yu. V. Gusev, G.A. Vilkovisky and Zhytnikov, J.Math.Phys. 35 3525;3543 (1994); Nucl.Phys. B 439561 (1995); A.O. Barvinsky, A.G. Mirzabekian and V.V. Zhytnikov, gr-qc/9510037.

[15] E. Elizalde, A.G. Jacksenaev, S.D. Odintsov, I.L.Shapiro, Class.Quant.Grav. 121385 (1995); Phys.Lett. 328B 297 (1994); E.Elizalde, S.D. Odintsov and I.L. Shapiro, Class.Quant.Grav. 111607 (1994).

[16] I. Antoniadis, J. Iliopoulos and T.N. Tomaras, Nucl.Phys. B261157 (1985);

[17] I.L. Shapiro and H. Takata, Phys.Lett. 361 B, 31 (1996).

[18] J.D. Bekenstein, Ann.Phys. 82,535,(1974).

[19] I.L. Shapiro, Class.Quant.Grav. 14, 391 (1997).

[20] I.L. Shapiro and A.G. Jacksenaev, Phys.Lett. 324B, 284 (1994).

[21] B.L. Voronov, P.M. Lavrov and I.V. Tyutin, Sov.J.Nucl.Phys. 36 (1982) 498; I.V. Tyutin, Sov.J.Nucl.Phys. 35 (1982) 125.

[22] K.S. Stelle, Phys.Rev. 16D, 953 (1977).

[23] I.L. Buchbinder, Theor.Math.Phys. 61, 393 (1984).

[24] I.L. Buchbinder, S.D. Odintsov and I.L. Shapiro, Effective Action in Quantum Gravity (IOP, Bristol, 1992).

[25] H.-J. Schmidt, A new duality transformation for fourth-order gravity, hep-th/9703002, Gen.Rel.Grav., to be published. 
[26] J.D. Barrow, S. Cotsakis, Phys.Lett. 214B, (1988) 515.

[27] S. Paneitz, A Quartic Conformally Covariant Differential Operator for Arbitrary PseudoRiemannian Manifolds, MIT preprint, 1983.

[28] T.P. Branson, Comm.Part.Diff.Equations 7 (1982) 393; Math.Scand. 57 (1985) 293.

[29] T.P. Branson, Commun.Math.Phys. 178 (1996) 301.

[30] J. Erdmenger, hep-th/9704108.

[31] M.J. Duff, Nucl.Phys. B125, (1977) 334. 